\title{
Arthroscopic partial capsulotomy for exposure and treatment of hip disease
}

\author{
ZE ZHUANG $^{1 *}$, YUXIAN CHEN $^{1 *}$, KISHOR CHHANTYAL $^{2}$, RONGHAN HE $^{1}$, \\ TANGZHAO LIANG ${ }^{1}$ and DEHAI SHI ${ }^{1}$ \\ Departments of ${ }^{1}$ Joint Surgery and Orthopedic Trauma, and ${ }^{2}$ Spine Surgery, \\ The Third Affiliated Hospital of Sun Yat-sen University, Guangzhou, Guangdong 510630, P.R. China
}

Received October 31, 2017; Accepted May 15, 2018

DOI: $10.3892 /$ etm.2018.6455

\begin{abstract}
Hip arthroscopy is an effective method for the diagnosis and treatment of hip joint pathologies. However, gaining access to the central and peripheral compartments is challenging. The present study aimed to assess the advantages of using an arthroscopic extra-capsular approach and partial capsulotomy for access and subsequent management of hip diseases. Patients subjected to hip arthroscopy by partial capsulotomy for exposure and treatment of hip diseases between February 2012 and February 2016 were retrospectively analyzed. A total of 32 patients, including 19 males and 13 females, aged 19-48 years (median age, 36 years), had undergone the procedure. Firstly, the distal anterior lateral and anterolateral arthroscopic approach with blunt dissection was performed. Subsequently, a T-shaped partial capsulotomy was established to achieve adequate exposure. The shaver, radiofrequency probe and tissue penetrating suture grasper were then inserted to perform procedures including debridement of the synovium, suturing of the glenoid labrum. During surgery, a probe hook was used to push the capsule section limbs or pull the sutures placed on the capsule section limbs to improve exposure. For patients with pre-operative anterior instability, ligamentous laxity or acetabular dysplasia capsules were sutured to finish capsule closure. The pre-operative and post-operative Visual Analogue Scale (VAS) score and modified Harris hip score (MHHS) were used to assess the effectiveness of the procedure. No obvious post-operative complications were encountered. The mean follow-up time was 22.4 months (range, 18-32 months) and 31 patients completed the
\end{abstract}

Correspondence to: Dr Dehai Shi, Department of Joint Surgery and Orthopedic Trauma, The Third Affiliated Hospital of Sun Yat-sen University, 600 Tianhe Road, Guangzhou, Guangdong 510630, P.R. China

E-mail: shidehaisums@163.com

*Contributed equally

Key words: hip arthroscopy, capsulotomy, exposure, hip disease follow-up, while 1 patient was lost to follow-up. Compared with the pre-operative score, the MHHS was significantly increased $(66.2 \pm 6.0$ vs. $82.6 \pm 5.2 ; \mathrm{P}<0.05)$ and the VAS score was significantly decreased $(6.5 \pm 1.1$ vs. $1.2 \pm 0.7 ; \mathrm{P}<0.05)$ at the end of the follow-up. In conclusion, arthroscopic partial capsulotomy provides access to the peripheral and central compartments of the hip and is a relatively simple technique that is easy to master for surgeons with limited experience in hip surgery.

\section{Introduction}

Hip arthroscopy is an effective method for diagnosing and treating hip joint diseases. Its indications include acetabular impingement, hip synovial chondromatosis, glenoid labrum injury, cartilage injury, hip joint infection and hip pain of unknown origin. Its contraindications are joint stiffness, superficial infection and heterotopic ossification $(1,2)$. Furthermore, hip arthroscopy is a technically demanding procedure and difficult for surgeons to learn due to the thick subcutaneous fat layer and muscles surrounding the hip joint, the high level of congruency between the acetabulum and femoral head, and the special instruments required to perform the procedure. Conventional methods require precise hip puncture and adequate penetration of the capsule, which is relatively difficult to perform due to direct access to the central compartment being hindered by the ball-and-socket morphology of the joint. Therefore, the risk of chondral scuffing and labral penetration is high. During hip arthroscopy, the operating space is available for the surgical manipulations is insufficient, which may lead to inadequate treatment of the lesions (3).

Arthroscopic capsulotomy may be utilized to expose lesions in the hip joint and two methods are available to perform this (4). One is the commonly used interportal capsulotomy, in which the capsule is cut between the two portals. The length of the interportal capsulotomy varies depending on the individual surgeon's personal preference (5). Using this method, most lesions in the central compartment may be sufficiently exposed, while the exposure of lesions which lie distal to the femoral head is poor. The other method is T-shaped capsulotomy, which was first described by Horisberger et al (6) in 2009. This method 
comprises the generation of a space outside of the capsule, followed by a T-shaped capsulotomy along the iliofemoral ligament perpendicular to the intertrochanteric line. This is often referred to as an 'outside-to-inside' technique, which may provide adequate exposure to visualize the peripheral compartment, as well as increased room for instrument maneuverability. Compared with other joint puncture techniques, the ability to perform this technique may be easily acquired by most surgeons to achieve a satisfactory exposure and outcome. Certain improvements to the method described by Horisberger et al (6) were made, including suturing the capsule and implementing different capsular management strategies $(4,7)$. The present study aimed to describe and evaluate this technique.

\section{Patients and methods}

Patient selection. All patients who underwent hip arthroscopy at the Third Affiliated Hospital of Sun Yat-sen University (Guangzhou, China) between February 2012 and February 2016 were considered eligible for the study and were retrospectively analyzed, excluding those with infective hip arthritis. A total of 32 patients (18 cases with the left hip and 14 cases with the right hip affected), including 19 males and 13 females, aged between 19 and 48 years with a median age of 36 , were included in the present study. The cohort comprised 15 cases of hip synovitis, 9 cases of hip impingement syndrome and 8 cases of hip joint synovial chondromatosis.

Pre-operative bilateral anteroposterior hip X-ray (AP view), frog leg view and Lequesne false profile X-ray images were captured $(8,9)$. Computed tomography of the hip joint was also performed to analyze the morphology of the femoral head and the acetabulum. Patients who were suspected of having labrum or cartilage damage underwent magnetic resonance imaging (MRI) examination.

Surgical technique: Extracapsular arthroscopic approach. Standard equipment, including specific cannula, shavers, a radiofrequency probe, 30 and $70^{\circ}$ viewing arthroscope (Smith \& Nephew plc, London, UK), a C-arm fluoroscope and a traction table were prepared. The patients were placed in the supine position on the orthopaedic traction bed with the operating leg immobilized at $20^{\circ}$ hip flexion. The other, unaffected leg was fixed with a leg holder and kept away from the operation field. A well-padded bolster was placed under the perineum to prevent injury to the genitalia and pudendal nerve.

The great trochanter and the anterior superior iliac spine were marked with a sterile pen. The anterolateral portal (ALP) was placed 1-2 cm proximal to the tip of the greater trochanter and the AP view by $\mathrm{C}$-arm was used to monitor the arthroscope (blunt trocar) to reach the extracapsular position of the femoral head-neck junction, just outside of the hip capsule (Fig. 1). The distal anterolateral accessory portal was set up ventrally and distal to the anterior border of the trochanter. The blunt trocar was inserted into the femoral head-neck junction under AP view. Subsequently, the radiofrequency probe or shaver was placed into the femoral head-neck junction through the trocar (Fig. 2). The adipose tissues between the iliocapsularis tendon medially and the gluteus minimus tendon laterally were removed under direct arthroscopic visualization and the white capsular fibers were identified. The midanterior portal was established if required. Capsulotomy was performed along the fiber direction of the iliofemoral ligament. Partial cleaning of the joint capsule near the acetabulum was performed. Probe hook may also be used to improve the exposure by pushing joint capsules to both sides. If more exposure was required, the medial or the lateral limbs of the capsulotomy were retracted by pulling the sutures placed on the section limbs. In patients who required labrum repair or femoral head molding, a transverse cut along the acetabular rim was performed. The capsulotomy was performed parallel to the femoral neck to the labrum, and limited proximal to the zona orbicularis to avoid injury to the terminal branch of the lateral femoral circumflex artery (Fig. 3).

On a standard traction table, lower limb traction was applied to facilitate the insertion of the arthroscope into the central compartment. The acetabular labrum was located by identifying the reflex portion of the rectus femoris muscle that was visible on arthroscopy. Subsequently, diagnostic visualization and treatment of any pathology in the central compartment was performed. Following procedures including removal of synovial loose bodies, debridement of delaminated cartilage, hip synovial biopsy (Fig. 4) and repair of the labrum, the traction was removed after the procedures in the central compartments were completed so as to reduce injury to the nerve. The arthroscope was then moved to the peripheral compartment of the hip and the synovium was cleaned to observe the head-neck junction. For cam-type femoroacetabular impingement patients, the bump of the cam lesion was resected under the $\mathrm{C}$-arm to achieve an impingement-free range of movement (Fig. 5). This was performed carefully to prevent femur neck fracture and injury to the ascending branch of the circumflex femoral artery, which supplies blood to the femoral head.

In addition, excessive joint capsulotomy was avoided and the capsule was not routinely sutured. However, for certain patients with pre-operative anterior instability, ligamentous laxity or acetabular dysplasia, a midanterior portal was established. The hip was flexed to $30^{\circ}$ and a sharp tissue penetrating suture grasper (BirdBeak; Arthrex GmbH, Munich, Germany) and Suture Lasso (Smith \& Nephew plc) were used. The Suture Lasso was used to pierce the proximal side of the capsulotomy and a looped No. 2 nylon suture lasso was then shuttled through the tissue. The grasper was used to retrieve the nylon lasso through the distal side of the capsulotomy. A further No. 2 suture was shuttled into the lasso and pulled out. Finally, both capsular limbs were sutured to achieve a side-to-side stitch, with the knot tied outside of the capsule, to perform the capsule closure (10). While Horisberger et al (6) did not surture the capsule.

Post-operative rehabilitation. Patients were routinely prescribed oral indomethacin for prophylaxis of heterotopic ossification for 4 weeks. Those who received femoroplasty were ambulated with crutches for 4 weeks as a precautionary measure to prevent fracture of the femoroplasty site. However, patients diagnosed with synovial chondromatosis and hip synovitis were allowed immediate weight bearing 

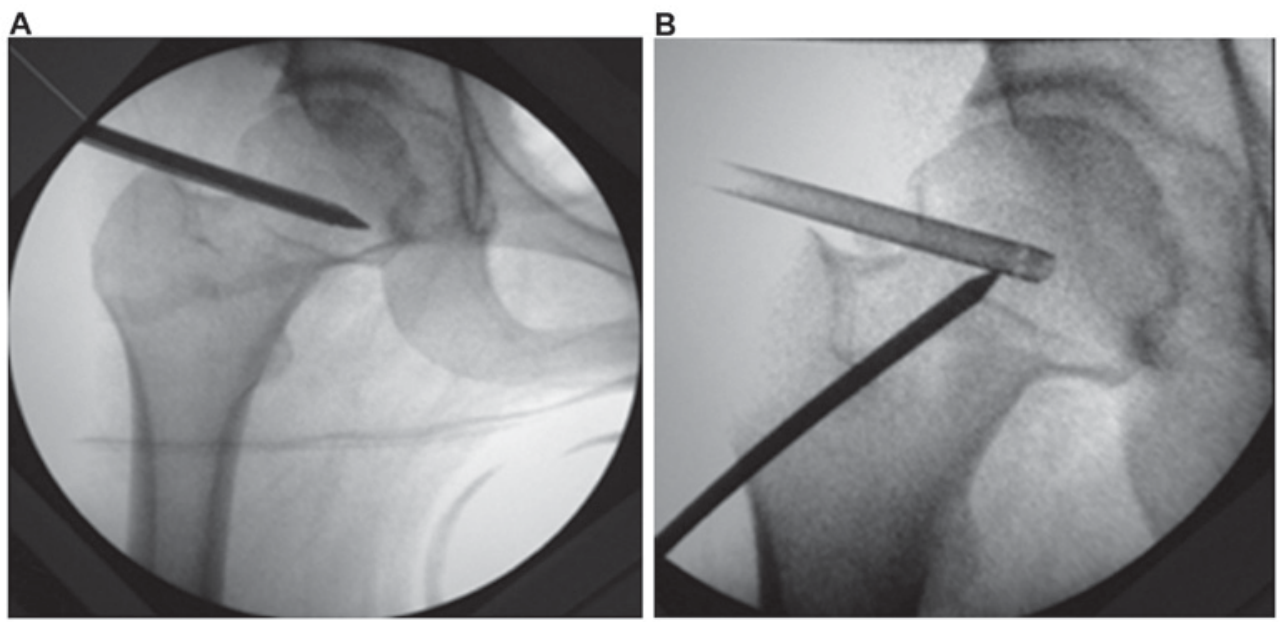

Figure 1. Location of the joint capsulotomy range visualized by C-arm fluoroscopy. (A) Using the anterolateral approach, the trocar was punctured into the head-neck junction and the arthroscopy cannula was inserted. (B) Using the distal anterolateral accessory portal, the trocar was placed in order to converge with the cannula, using the anterolateral approach.

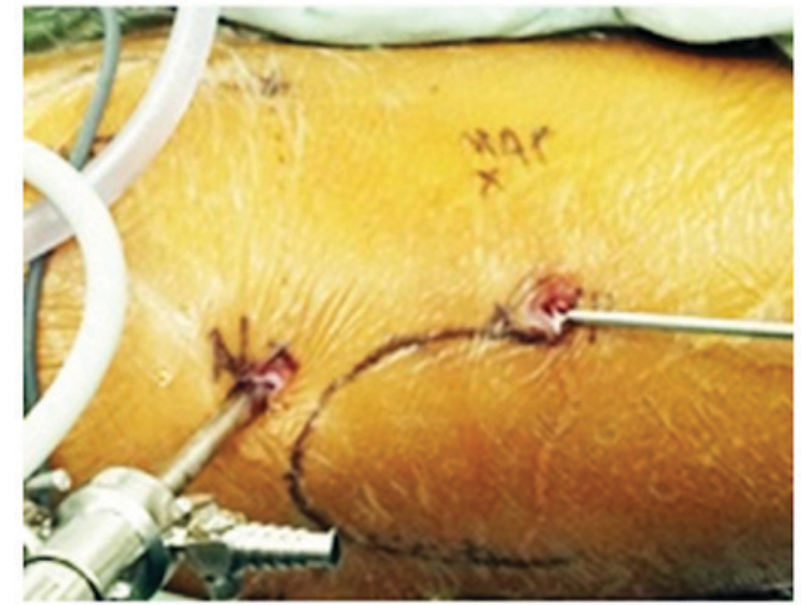

Figure 2. Substantial surface view displaying the location of the arthroscopic approach.

post-operatively. After 12 weeks, patients resumed their daily activities, and gradually took up low-impact physical exercise, including jogging and climbing stairs. After 6 months, the patients were allowed to gradually return to sports (11-13).

Pre-operation and at the last follow-up activity and functional levels were recorded using the modified Harris hip score (MHHS) and The Visual Analogue Scale (VAS). MHHS is widely used to assess the outcomes of hip arthroscopic procedures (14-17). Patients were followed-up with telephone interviews. The details of MHHS are listed in Table I. Unlike the Harris hip score, the range of motion and absence of deformity were not included in the MHHS. In addition, the VAS is a system that measures the intensity of pain.

Statistical analysis. Statistical analysis was performed using SPSS 21.0 (IBM Corp., Armonk, NY, USA). MHHS and VAS score were expressed as the mean \pm standard deviation, and compared using a wilcoxon signed rank test. $\mathrm{P}<0.05$ was considered to indicate a statistically significant difference.

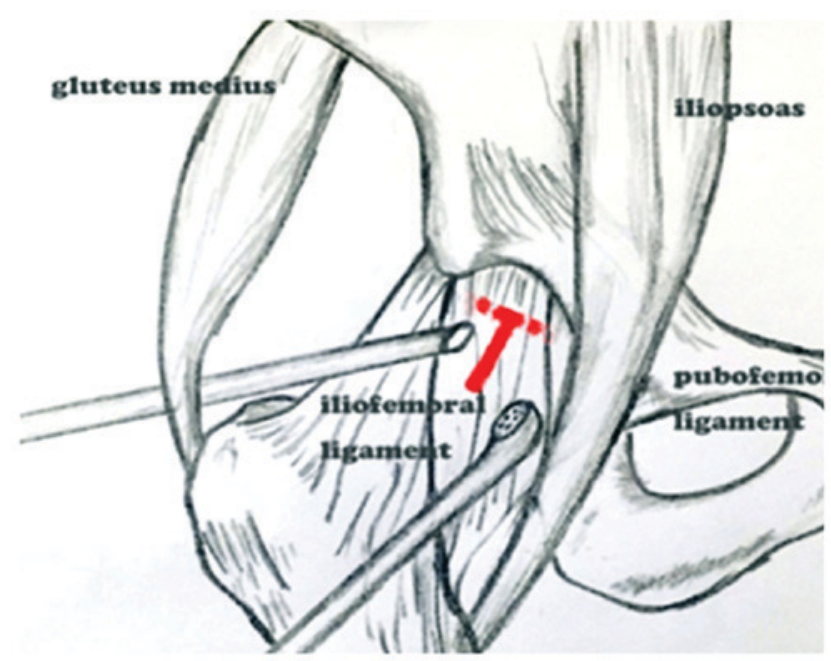

Figure 3. Schematic indicating the operating range of the arthroscopic partial capsulotomy. The red solid line demonstrates the range of capsulotomy and the red dotted line indicates the range to be cut further depending on the requirements of surgery. The upper and lower instruments depicted in the diagram are the arthroscopy and the condensation knife, respectively.

\section{Results}

Complications. No complications including joint infection, perineal skin compression, heterotopic ossification or injury of the lateral femoral cutaneous nerve, sciatic nerve or pudendral nerve were observed peri-operatively.

MHHS and VAS results. The follow-up duration was 18-32 months, with a mean of $22.4 \pm 2.2$ months. Of the total cohort, 31 patients were available for follow-up, while 1 patient was lost to follow-up. MHHS was $66.2 \pm 6.0$ and the Visual Analogue Score (VAS) was 6.5 \pm 1.1 . At the end of the follow-up period, the MHHS was $82.6 \pm 5.2$ and the VAS was 1.2 \pm 0.7 . While the modified Harris score was significantly increased compared with the pre-operative score $(\mathrm{P}<0.05)$, the VAS score at the end of the follow-up was significantly lower than that at the pre-operative stage $(\mathrm{P}<0.05$; Table II $)$. 


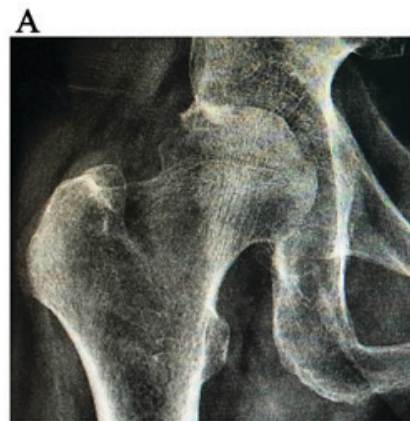

$\mathrm{C}$

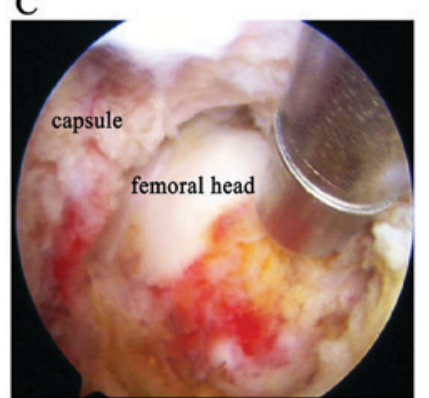

B

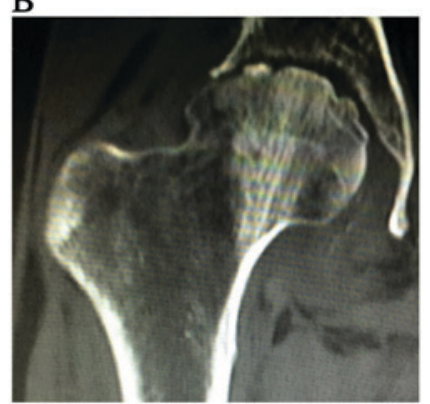

D

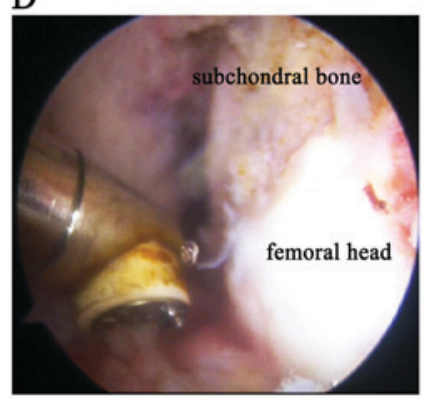

Figure 4. A 48-year-old male presented with right hip pain for $>4$ months. The patient was admitted to hospital on suspicion of a tuberculosis infection, and a hip arthroscopy examination was performed. (A) X-ray and (B) CT scans demonstrated a narrow hip joint space, with cartilage damage. (C and D) intraoperative endoscopy images were obtained. (C) Following capsulotomy the femoral head was revealed. (D) The patient's femoral head cartilage was extensively exfoliated and the underlying subchondral bone was exposed.

\section{Discussion}

Anatomically, the hip capsule is reinforced by the iliofemoral, pubofemoral and ischiofemoral ligament. The iliofemoral ligament, which tightens with hip extension, is the most important and strongest ligament in the hip. It is divided into two branches and forms an inverted Y-shape anteriorly, and the flat plane in front of the two branches separates the iliocapsularis muscle and the gluteus minimus. The pubofemoral ligament is on the medial and inferior side of the hip joint, and the ischiofemoral ligament is on the posterior side (18). The hip joint may be divided into a central compartment and a peripheral compartment. By identification of the reflex portion of the rectus femoris muscle, the location of the acetabular labrum may be determined. The insertion of the rectus femoris on the pelvis starts from the upper edge of the acetabulum at the 12 o'clock position, and the blood supply to the femoral head is mainly from the medial femoral circumflex artery, which arises near the base of the femoral neck, so that partial capsulotomy is less likely to affect the blood supply of the femoral head.

In the conventional method, due to the prominent anterior acetabular wall and the tough capsule surroundings, sufficient lower limb traction must be applied first to enlarge the joint space. The surgeon may then use a needle to puncture the central compartment under fluoroscopic guidance. Anitinol guide wire is passed into the joint through the needle and metal tubes of different diameters are consecutively placed to expand the tunnel. Following this, the arthroscopic tube may be inserted into the joint via the expanded tunnel. An interportal capsulotomy may be used to enlarge the area for operation. Finally, the arthroscope and a shaver may be placed
A

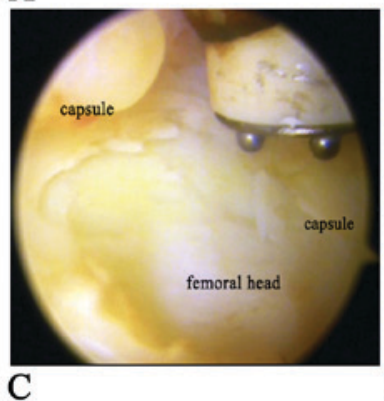

B

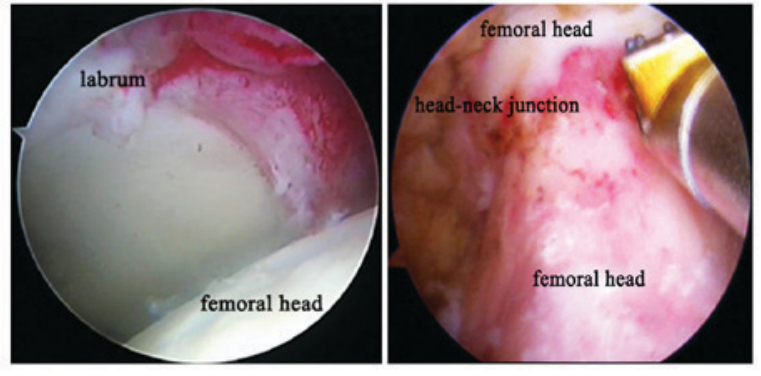

E
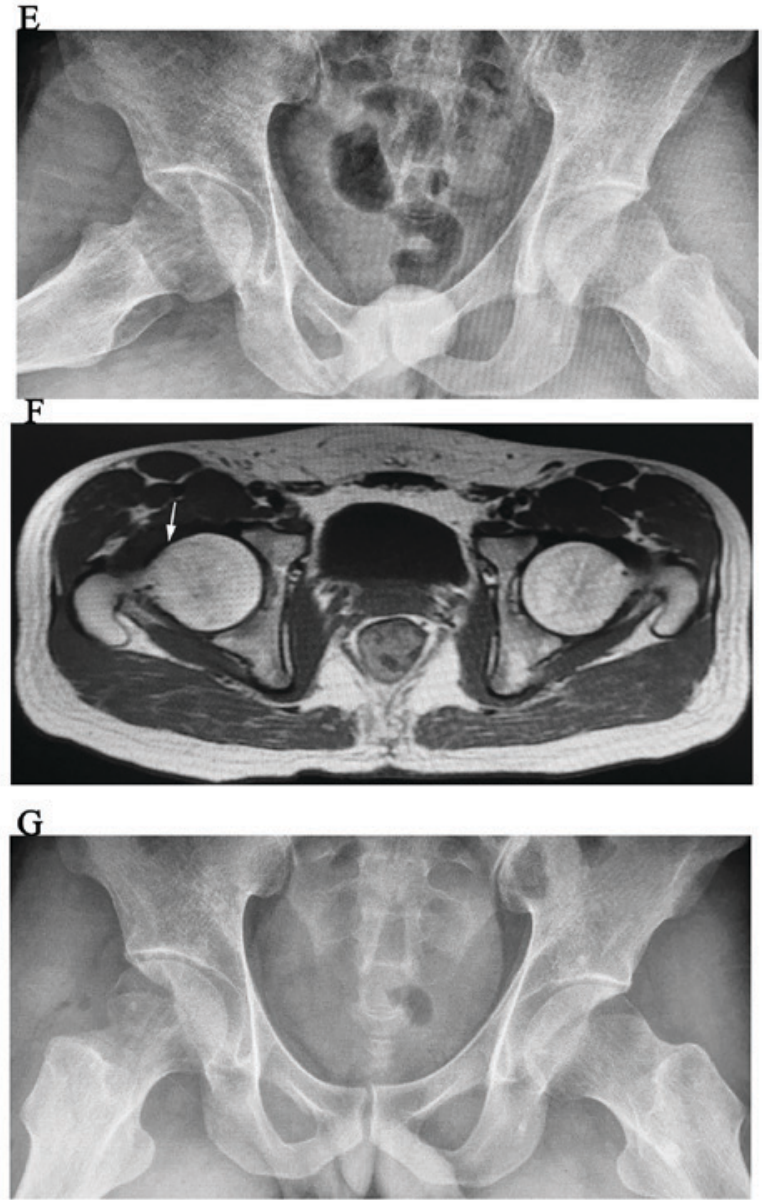

Figure 5. Once the pre-capsular adipose tissue was removed with an electrode and capsulotomy was performed, the femoral head and the acetabular labrum were exposed. (A-D) intraoperative endoscopy images were obtained. (A) Following partial capsulotomy, the femoral head below was visible. (B) By performing distraction of the lower limb, the arthroscopy slid into the central compartment. The lower right side was the femoral head. (C) The upper left side is the labrum and the lower right side is the femoral head. (D) The head-neck junction of the femoral head. (E) Pre-operative X-ray and $(\mathrm{F})$ pre-operative magnetic resonance image demonstrated that there was more bulging on the right side of the femoral head than the left side (as indicated by the arrow). (G) post-operative X-ray results. All images were obtained from a 28-year-old male patient diagnosed with cam-type femoroacetabular impingement. 
Table I. Modified-Harris hip scoring system.

Points

\begin{tabular}{l} 
Parameter \\
\hline I. Pain \\
None \\
Slight or occasional, no activity com \\
Mild, no effect on average activity, \\
non-steroidal anti-inflammatory agent \\
Moderate, some average activity lim \\
occasional narcotic use \\
Marked, serious activity limitations \\
Totally disabled, bedridden
\end{tabular}

II. Function

44

44

40

30

20

10

0

47

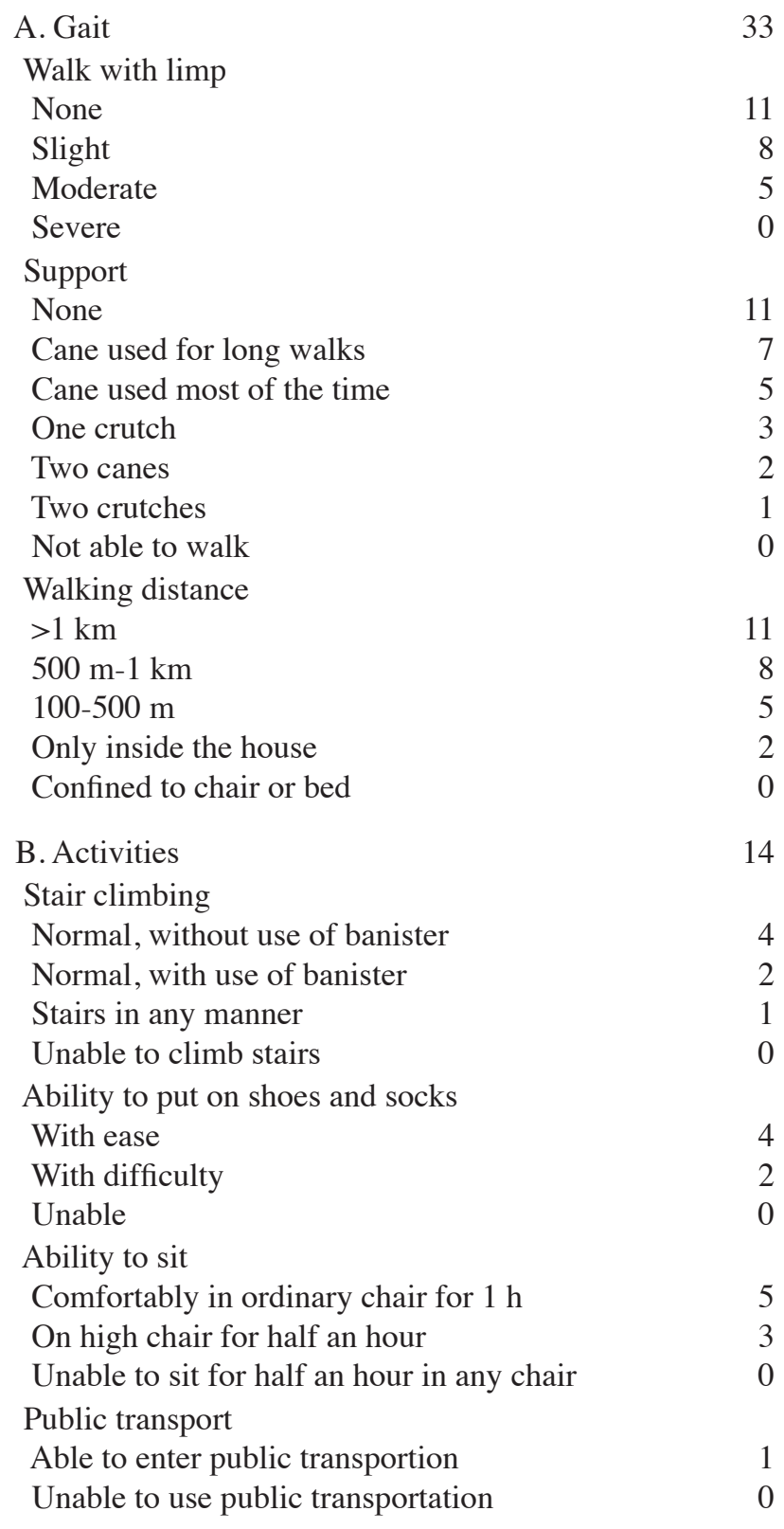

into the joint. A major drawback of this technique is that it may be difficult to perform for less experienced surgeons. Furthermore, repeated puncturing may lead to articular cartilage or labrum injury, as well as prolonged operation and traction time. Prolongation of the traction time may also lead to nerve injury and continued pressure between the perineum column and the skin may cause skin compression (19). In addition, certain lesions lying distal to the femoral head may not be sufficiently visualized due to the overlying capsule and the relatively narrow operative area. Due to the combination of these factors, treatment using the conventional method may be insufficient, resulting in residual deformity and patient dissatisfaction regarding the outcome.

Arthroscopic partial capsulotomy is an 'outside to inside' method, which differs from the conventional puncture method. Initially, the surgeon follows a Hueter approach at the front of the joint, and once the partial capsulotomy has been performed, the arthroscope may be inserted into the central compartment from the outside to the inside under traction.

This method was first described by Horisberger et al (6), and in the present study, certain modifications have been applied. First, no adrenalin solution was used to expand the joint capsule. In the beginning, punctures were made directly outside the capsule. However, needle puncture into the joint capsule is not necessary. By avoiding repeated joint puncture, the operative duration may be reduced; furthermore, the chances of labrum damage are reduced if performed by a less experienced or unskillful surgeon. Furthermore, the transverse cut along the acetabular rim is rarely required, as partial cleaning of the joint capsule near the acetabulum is usually performed at our department. By putting a probe hook to push the section limbs of the capsule or pulling the sutures placed on the section limbs of the capsule and passive rotation and distraction of the lower limb, superior exposure was achieved in most cases of the present study. This presents a difference from the method described by Horisberger et al (6), which relied on the Wissinger rod or Hohmann retractor over the proximal anterolateral portal. In addition, the T-shaped cut was performed only in patients who required labrum repair or femoral head-neck molding.

In addition, Horisberger et al (6) reported that, out of a total of 105 hips, dysesthesia/hypesthesia of the pudendal and lateral cutaneous femoral nerves occurred in 9 cases. In the present study, not a single case of lateral cutaneous nerve complications was encountered. Injury to this nerve, which lies just below the inguinal ligament and in front of the Sartorius and tensor fascia latae (20), may occur during portal establishment. In order to reduce the risk of nerve injury in the present study, following the incision of the skin, the vascular forceps were used to carefully separate the subcutaneous tissue, and the trocar was then slowly inserted. Finally, Horisberger et al (6) did not report whether the capsule was repaired after capsulotomy. In the present study, those patients with pre-operative anterior instability, ligamentous laxity or acetabular dysplasia, the joint capsule was repaired with absorbable sutures.

The partial capsulotomy used in the present study has several advantages. One major benefit is that it prevents possible iatrogenic cartilage and labrum damage from repeated puncturing of the capsule, as all of the manipulations are completed under direct visualization. This may also save time if the operation is performed by a surgeon who is less experienced in hip surgery. Only when partial capsulotomy is performed, the surgeon may easily enter the joint, has a wider operative space and improved visualization of the lesion, and 
Table II. Comparison between pre- and post-arthroscopy using MHHS and VAS.

\begin{tabular}{lcccr}
\hline & Total cases & Pre-operative score & Post-operative score & P-value \\
\hline VAS & 31 & $6.5 \pm 1.1$ & $1.2 \pm 0.7$ & $P<0.05$ \\
MHHS & 31 & $66.2 \pm 6.0$ & $82.6 \pm 5.2$ & $P<0.05$ \\
\hline
\end{tabular}

MHHS, Modified Harris Hip Score; VAS, visual analogue scale.

is able to conveniently fix the bone deformity. Furthermore, the traction time and strength may be reduced, as it is only used after placing the portals and performing the capsulotomy. Thus, the risk of sciatic or pudendal nerve injury is reduced.

In addition, partial capsulotomy does not require any special surgical instruments, including the hollow metal tube or nitinol guide wire. The puncture method is also relatively simple, and learning surgeons may quickly master this method. Thaunat et al (21) regarded capsulotomy as a simple and easy technique to master, which may be performed using a $30^{\circ}$ optic system, with the possibility of reduction in the force and duration of traction, and with a lesser risk of labral or chondral damage.

A shortcoming is that arthroscopic partial capsulotomy inevitably causes damage to the ligaments of the hip joint and part of the joint capsule. Indiscriminate capsulotomy should also be avoided in case of iatrogenic hip pain and instability after arthroscopy, particularly in patients with potential joint laxity and developmental hip dysplasia (22). It is controversial whether the capsule should be repaired after capsulotomy. Domb et al (23) performed a systematic review of $\sim 4,000$ cases of hip arthroscopic surgeries that used various degrees of capsule resection treatment of femoroacetabular impingement without patching or overlapping and identified no case of iatrogenic instability. Strickland et al (24) reported that repaired and unrepaired capsulotomy sites progressed to healing with a continuous appearance on MRI by 24 weeks post-operatively. Dippmann et al (25) performed a multicenter study to compare capsular closure with non-capsular closure during hip arthroscopy in Danish patients with femoroacetabular impingement, and concluded that whether the hip capsule should be closed during hip arthroscopy or not should be decided based on the biomechanical pre-conditions of the patients.

No general consensus has been reached regarding whether the capsule must be repaired, but undoubtedly, excessive joint capsulotomy should be avoided. According to our experience, which is based on individual situations with specific patients, capsular repair may be recommended for patients with pre-operative anterior instability, capsular hyperlaxity or hip development dysplasia. An anatomic side-to-side repair of the capsule from the distal to the proximal end may restore the native tension and anatomy of the iliofemoral ligament. However, for certain patients with adhesive capsulitis or limited external rotation prior to surgery, capsular repair may not be necessary.

In addition, injury to the circumflexae femoris lateralis artery may be avoided by paying full attention while removing the adipose tissues between the gluteus minus laterally and the iliocapsular medially. Excessive resection during osteochondroplasty should also be avoided to prevent femoral neck fractures (26).

Of note, the present study had several limitations. The number of cases included in the study were limited. Furthermore, the present study assessed only one treatment group, without an additional treatment group for comparison. The VAS/MHHS score were also not assessed at the same time-point for all patients. Future studies should therefore address these issues and include additional outcomes for patient characteristics and clinicopathological data.

In conclusion, the arthroscopic partial capsulotomy technique presented in the present study provides an alternative option for exposure and treatment of hip joint disease. This method yielded satisfactory clinical outcomes and without any significant complications, and is easy to master for surgeons with limited experience in hip arthroscopy.

\section{Acknowledgements}

Not applicable.

\section{Funding}

The present study was financially supported by the Guangdong Medical Science and Technology Research Fund (grant no. A2016066), the Guangdong Traditional Chinese Medicine Bureau Research Fund (grant no. 20181061) and the Guangdong Medial Scientific Research Fund (grant no. A2015115).

\section{Availability of data and materials}

The datasets used and/or analyzed during the current study are available from the corresponding author on reasonable request.

\section{Authors' contributions}

$\mathrm{ZZ}$ and DS conceived the study. ZZ performed the literature search and wrote the manuscript. YC contributed to the interpretation of data and review of the manuscript. KC contributed to the analysis and interpretation of data, as well as language editing. RH and TL collected and assembled the data. All authors have read and approved the final manuscript.

\section{Ethics approval and consent to participate}

Not required due to the retrospective nature of the study. 


\section{Patient consent for publication}

Patients have provided consent for publication.

\section{Competing interests}

The authors declare that they have no competing interests

\section{References}

1. McCarthy JC and Lee JA: Hip arthroscopy: Indications, outcomes, and complications. Instr Course Lect 55: 301-308, 2006.

2. Sampson TG: Complications of hip arthroscopy. Clin Sports Med 20: 831-835, 2001.

3. Mehta N, Chamberlin P, Marx RG, Hidaka C, Ge Y, Nawabi DH and Lyman S: Defining the learning curve for hip arthroscopy: A threshold analysis of the volume-outcomes relationship. Am J Sports Med 46: 1284-1293, 2018.

4. Ekhtiari S, de Sa D, Haldane CE, Simunovic N, Larson CM, Safran MR and Ayeni OR: Hip arthroscopic capsulotomy techniques and capsular management strategies: A systematic review. Knee Surg Sports Traumatol Arthrosc 25: 9-23, 2017.

5. Bedi A, Galano G, Walsh C and Kelly BT: Capsular management during hip arthroscopy: From femoroacetabular impingement to instability. Arthroscopy 27: 1720-1731, 2011.

6. Horisberger M, Brunner A and Herzog RF: Arthroscopic treatment of femoroacetabular impingement of the hip: A new technique to access the joint. Clin Orthop Relat Res 468 : 182-190, 2010

7. Forster-Horvath C, Domb BG, Ashberg L and Herzog RF: A method for capsular management and avoidance of iatrogenic instability: Minimally invasive capsulotomy in hip arthroscopy. Arthrosc Tech 6: e397-e400, 2017.

8. Clohisy JC, Carlisle JC, Beaulé PE, Kim YJ, Trousdale RT, Sierra RJ, Leunig M, Schoenecker PL and Millis MB: A systematic approach to the plain radiographic evaluation of the young adult hip. J Bone Joint Surg Am 90 (Supp 4): 47-66, 2008.

9. Hellman MD, Mascarenhas R, Gupta A, Fillingham Y, Haughom BD, Salata MJ and Nho SJ: The false-profile view may be used to identify cam morphology. Arthroscopy 31: 1728-1732, 2015.

10. Camp CL, Reardon PJ, Levy BA and Krych AJ: A simple technique for capsular repair after hip arthroscopy. Arthrosc Tech 4: e737-e740, 2015.

11. Grzybowski JS, Malloy P, Stegemann C, Bush-Joseph C, Harris JD and Nho SJ: Rehabilitation following hip arthroscopy-a systematic review. Front Surg 2: 21, 2015.

12. Enseki KR and Kohlrieser D: Rehabilitation following hip arthroscopy: An evolving process. Int J Sports Phys Ther 9: 765-773, 2014.

13. Rath E, Sharfman ZT, Paret M, Amar E, Drexler M and Bonin N: Hip arthroscopy protocol: Expert opinions on post-operative weight bearing and return to sports guidelines. J Hip Preserv Surg 4: 60-66, 2017
14. Lavini F, Renzi-Brivio L, Aulisa R, Cherubino F, Di Seglio PL, Galante N, Leonardi W and Manca M: The treatment of stable and unstable proximal femoral fractures with a new trochanteric nail: Results of a multicentre study with the Veronail. Strategies Trauma Limb Reconstr 3: 15-22, 2008.

15. Harris WH: Traumatic arthritis of the hip after dislocation and acetabular fractures: Treatment by moldarthroplasty. An end-result study using a new method of result evaluation. J Bone Joint Surg Am 51: 737-755, 1969.

16. Byrd JW and Jones KS: Prospective analysis of hip arthroscopy with 2-year follow-up. Arthroscopy 16: 578-587, 2000.

17. Hung M, Hon SD, Cheng C, Franklin JD, Aoki SK, Anderson MB Kapron AL, Peters CL and Pelt CE: Psychometric evaluation of the lower extremity computerized adaptive test, the modified harris hip score, and the hip outcome score. Orthop J Sports Med 2: 2325967114562191, 2014.

18. Martin HD, Savage A, Braly BA, Palmer IJ, Beall DP and Kelly B: The function of the hip capsular ligaments: A quantitative report. Arthroscopy 24: 188-195, 2008.

19. Nakano N, Lisenda L, Jones TL, Loveday DT and Khanduja V: Complications following arthroscopic surgery of the hip: A systematic review of 36761 cases. Bone Joint J 99-B: 1577-1583, 2017.

20. Tomaszewski KA, Popieluszko P, Henry BM, Roy J, Sanna B, Kijek MR and Walocha JA: The surgical anatomy of the lateral femoral cutaneous nerve in the inguinal region: A meta-analysis. Hernia 20: 649-657, 2016.

21. Thaunat M, Murphy CG, Chatellard R, Sonnery-Cottet B, Graveleau N, Meyer A and Laude F: Capsulotomy first: A novel concept for hip arthroscopy. Arthrosc Tech 3: e599-e603, 2014.

22. Ranawat AS, McClincy M and Sekiya JK: Anterior dislocation of the hip after arthroscopy in a patient with capsular laxity of the hip. A case report. J Bone Joint Surg Am 91: 192-197, 2009.

23. Domb BG, Philippon MJ and Giordano BD: Arthroscopic capsulotomy, capsular repair, and capsular plication of the hip: Relation to atraumatic instability. Arthroscopy 29: 162-173, 2013.

24. Strickland CD, Kraeutler MJ, Brick MJ, Garabekyan T, Woon JTK, Chadayammuri V and Mei-Dan O: MRI evaluation of repaired versus unrepaired interportal capsulotomy in simultaneous bilateral hip arthroscopy: A double-blind, randomized controlled trial. J Bone Joint Surg Am 100: 91-98, 2018.

25. Dippmann C, Kraemer O, Lund B, Krogsgaard M, Holmich P Lind M, Briggs K, Philippon $M$ and Mygind-Klavsen B: Multicentre study on capsular closure versus non-capsular closure during hip arthroscopy in Danish patients with femoroacetabular impingement (FAI): Protocol for a randomised controlled trial. BMJ Open 8: e019176, 2018

26. Horner NS, Vikas K, MacDonald AE, Naendrup JH, Simunovic N and Ayeni OR: Femoral neck fractures as a complication of hip arthroscopy: A systematic review. J Hip Preserv Surg 4: 9-17, 2017.

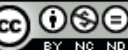

This work is licensed under a Creative Commons Attribution-NonCommercial-NoDerivatives 4.0 International (CC BY-NC-ND 4.0) License. 